\title{
Polyphyllin VII induces mitochondrial apoptosis by regulating the PP2A/AKT/DRP1 signaling axis in human ovarian cancer
}

\author{
LIUBIQI ZHAO ${ }^{1}$, ZONGYU LIU $^{1}$, XINYUE DENG ${ }^{2}$, JIABIN WANG $^{2}$, \\ LIANKUN SUN $^{2}$, LIMEI FAN ${ }^{1}$ and YONG ZHANG ${ }^{2}$ \\ ${ }^{1}$ Department of Gynecology and Obstetrics, The Second Hospital of Jilin University; \\ ${ }^{2}$ Department of Pathophysiology, Key Laboratory of Pathophysiology, Ministry of Education, \\ College of Basic Medical Sciences, Jilin University, Changchun, Jilin 130041, P.R. China
}

Received May 11, 2020; Accepted October 19, 2020

DOI: $10.3892 / o r .2020 .7879$

\begin{abstract}
Ovarian cancer is a gynecological malignancy with high mortality. Adjuvant therapy such as chemoradiotherapy inevitably leads to side effects and drug resistance. In recent years, traditional Chinese medicine has been widely studied for its safety, effectiveness, and unique pharmacological effects. Polyphyllin VII is an important component of Rhizoma paridis saponins, and has cytotoxic effects on many types of cancer cells. The aim of the present study was to evaluate the anti-tumor activity of polyphyllin VII in human ovarian cancer cells. Recent studies found that polyphyllin VII induces mitochondrial pathway apoptosis by increasing mitochondrial division, but the specific mechanism was unclear. The results of this study revealed that polyphyllin VII could effectively induce mitochondrial dysfunction, including increased mitochondrial division and reactive oxygen species (ROS) production. Notably, the mitochondrial location of dynamin-related protein 1 (DRP1) plays an important role in its function. In addition, polyphyllin VII enhanced the mitochondrial localization of DRP1 which is mediated by increased protein phosphatase 2A (PP2A) activity, and decreased AKT activity. A specific PP2A inhibitor, LB100, attenuated mitochondrial division and apoptosis in cells caused by polyphyllin VII, confirming the function of the PP2A/AKT pathway in polyphyllin VII treatment. Additionally, xenotransplantation experiments have also
\end{abstract}

Correspondence to: Dr Limei Fan, Department of Gynecology and Obstetrics, The Second Hospital of Jilin University, 218 Ziqiang Street, Nanguan, Changchun, Jilin 130041, P.R. China

E-mail: fanlimei1971@126.com

Dr Yong Zhang, Department of Pathophysiology, Key Laboratory of Pathophysiology, Ministry of Education, College of Basic Medical Sciences, Jilin University, 126 Xinmin Street, Changchun, Jilin 130021, P.R. China

E-mail: zyloulan@163.com

Key words: polyphyllin VII, PP2A, AKT, dynamin-related protein 1 , ovarian cancer confirmed the anti-tumor effect of polyphyllin VII in vivo. Therefore, interference of the mitochondrial translocation of DRP1 through PP2A/AKT pathway may be an attractive and effective therapeutic approach by polyphyllin VII in ovarian cancer. This may provide new strategies for polyphyllin VII in the clinical treatment of ovarian cancer.

\section{Introduction}

The majority of patients with ovarian cancer may suffer a relapse, and the development of platinum resistance results in great challenges to clinical treatment; thus, much effort has been placed into developing new drugs. Due to polyphyllin VII having pharmacological effects, such as anti-inflammatory (1), hemostatic and analgesic (2), immune regulation (3), and minor side effects, it has been widely used in clinical applications. In recent years, findings have shown that polyphyllin VII has obvious anti-cancer activity against ovarian cancer cells (4) prostate (5), gastric (6), nasopharyngeal (7), and colon cancer (8). Yang et al found that polyphyllin VII can regulate the expression of $\mathrm{Bcl}-2$ and $\mathrm{Bax}$, cause a decrease in mitochondrial membrane potential (MMP), and induce apoptosis in human erythroleukemia cell line K562 (9). Liu et al found in the study of the hepatocellular carcinoma HepaRG that polyphyllin VII can increase intracellular ROS production, decrease MMP, and accompany activation of the caspase pathway and the release of cytochrome $c$ from the cytoplasm to induce mitochondrial apoptosis (10). It is suggested that polyphyllin VII may cause mitochondrial dysfunction of tumor cells and induce apoptosis, although the specific mechanism of regulating mitochondrial function is not clear. Therefore, investigating the specific regulation mechanism of polyphyllin VII on mitochondrial function may provide some targeted evidence for the clinical treatment of ovarian cancer.

Mitochondria are not only the key organelles for intracellular energy production, but also the basic platform for cell signal transduction, playing a crucial role in cell signal transduction, cell proliferation, differentiation, autophagy and cellular immunity (11-13). Mitochondria are dynamic organelles, whose imbalance in division and fusion often leads to changes in mitochondrial structure and dysfunction (14). Although mitochondrial division often occurs in 
apoptosis, some scholars believe that division is an essential step in the process of apoptosis, although the specific regulatory mechanism of mitochondrial division and apoptosis is unclear. Mitochondrial fission is a multi-step process in which the recruitment of GTPase DRP1 on mitochondria plays a key role (15). During the process of cell apoptosis, BAX activation co-locates BAX and DRP1 at mitochondrial sites where division occurs (16), and DRP1 stably binds to the mitochondrial outer membrane, leading to mitochondrial fragmentation (17-19). Hasnat et al found that triptolide can mediate Drp1 translocation to the outer mitochondrial membrane, leading to increased mitochondrial division, accompanied by the release of cytochrome $c$ and activation of caspase-3, inducing L02 cell death in human liver cells (20). Li et al found that erucin could dephosphorylate DRP1 (Ser637) to mediate DRP1 mitochondrial translocation and induce mitochondrial division and apoptosis in human MDA-MB-231 and MCF-7 breast cancer cells (21). Therefore, the localization of DRP1 on mitochondria may mediate mitochondrial dynamics and mitochondrial pathway apoptosis, and this process is regulated by its phosphorylation level.

Protein phosphatase 2A (PP2A) is an important and ubiquitous serine threonine phosphatase and a tumor suppressor (22). PP2A is a critical negative regulator of tumorigenesis, and affects protein synthesis, cell proliferation, cell survival, cell migration, and invasiveness (23). PP2A is inactivated in a variety of malignancies, including breast (24), ovarian (25), cervical (26), and lung (27) cancer. Reactivating PP2A is an effective way to fight cancer (28). Zhang et al found that polyphyllin VII can inhibit the proliferation and invasion of cisplatin-resistant SGC7901/DDP cells by regulating the PP2A/AKT signaling axis (29). Kim et al found that Drp1 may be a direct substrate of AKT, whose activity is regulated by AKT, and their interaction induces phosphorylation of Drp1, leading to mitochondrial division (30). Therefore, we hypothesized that polyphyllin VII could regulate the phosphorylation of DRP1 and its mitochondrial localization by PP2A/AKT signaling axis.

In the present study, different concentrations of polyphyllin VII were used to treat human ovarian cancer cells to explore the association between changes in the mitochondrial localization of DRP1 and apoptosis through changes in DRP1 phosphorylation levels. The results showed that polyphyllin VII can significantly promote the translocation of DRP1 from the cytoplasm to mitochondria and increase the binding of pro-apoptotic proteins of the BCL-2 family to mitochondria, promoting the release of cytochrome $c$ into the cytoplasm. In addition, polyphyllin VII-induced changes in DRP1 localization are regulated by the dephosphorylation of DRP1 by PP2A/AKT. In summary, polyphyllin VII regulates mitochondrial division through the PP2A/AKT/DRP1 axis, and then promotes apoptosis, which provides a new idea for the clinical treatment of ovarian cancer with polyphyllin VII.

\section{Materials and methods}

Cell culture and reagents. Ovarian cancer cell lines A2780 and SKOV3 were purchased from the Chinese Academy of Medical Sciences and Peking Union Medical College (Peking, China) and cultured under the recommended conditions.
Polyphyllin VII with a purity of $98 \%$ or more was purchased from Sichuan Weikeqi Biotechnology Co., Ltd. The drug was dissolved in methyl sulfoxide (DMSO) (Sigma-Aldrich; Merck $\mathrm{KGaA}$ ) and stored at $-20^{\circ} \mathrm{C}$. The negative control group was the low concentration DMSO group. The final concentration of DMSO for all treatments was consistently less than $0.1 \%$. LB-100 was obtained from Apexbio.

MTT cell viability assay. Cells were seeded in 96-well plates and treated with different concentrations $(0,1,2,3 \mu \mathrm{M})$ of polyphyllin VII for $24 \mathrm{~h}$. The original medium was then removed and $0.5 \mathrm{mg} / \mathrm{ml} \mathrm{MTT} \mathrm{was} \mathrm{added} \mathrm{for} 4 \mathrm{~h}$ at $37^{\circ} \mathrm{C}$. Formazan crystals were dissolved in $150 \mu 1 \mathrm{DMSO}$ and absorbance was measured at a wavelength of $490 \mathrm{~nm}$.

Apoptosis analysis by flow cytometry. According to the manufacturer's instructions, A2780 and SKOV3 cells were stained with an Annexin V-FITC apoptosis kit (BD Bioscience) and analyzed using an Accuri C6 flow cytometer (BD Biosciences).

Assessment of mitochondrial depolarization. According to the manufacturer's instructions, pre-treated A2780 and SKOV3 cells were suspended in $1 \mathrm{ml}$ of complete medium containing $10 \mu \mathrm{g} / \mathrm{ml}$ of JC-1 (Beyotime) at $37^{\circ} \mathrm{C}$ for $30 \mathrm{~min}$. The cells were analyzed on an Accuri C6 flow cytometer (BD Biosciences).

Intracellular ROS measurements. To detect intracellular hydrogen peroxide levels, 2,7-dichlorodihydrofluorescein diacetate (DCFH-DA; Sigma-Aldrich; Merck KGaA) was used to measure intracellular ROS levels. Cell fluorescence was measured using an Accuri C6 flow cytometer (BD Biosciences).

Mitochondrial isolation. A Mitochondria Isolation Kit (Invent Biotechnologies) was used to extract mitochondria as directed by the manufacturer's protocol.

Western blot analysis. The A2780 and SKOV3 cells were lysed with $120 \mu \mathrm{l}$ of RIPA buffer (Beyotime). Cell lysates were sonicated for $30 \mathrm{sec}$ under ice and lysed at $4^{\circ} \mathrm{C}$ for $45 \mathrm{~min}$. The cells were lysed by centrifugation at $3,000 \mathrm{x} g$ for $15 \mathrm{~min}$, and the supernatant was used to determine the protein concentration using Bio-Rad kit (Pierce Biotechnology Inc.) and the samples were boiled for $5 \mathrm{~min}$. The cell lysates (10-20 $\mu \mathrm{g}$ ) were resolved on $12 \%$ SDS-polyacrylamide gels and transferred to polyvinylidene fluoride (PVDF) membranes. The membranes were blocked with $5 \%$ skim milk for $1 \mathrm{~h}$, and incubated with primary antibodies overnight at $4^{\circ} \mathrm{C}$. The following day, membranes were washed with PBST and incubated with horseradish peroxidase-conjugated secondary antibodies at 1:2,000 dilution for $1 \mathrm{~h}$ at room temperature. After washing the membranes with PBST, immunodetection was performed using ECL reagent (Thermo Fisher Scientific) and visualized using a Syngene Bio Imaging (Synoptics). The primary antibodies used were anti- $\beta$-actin (1:1,000 dilution), anti-cleaved caspase-3 (1:1,000 dilution), anti-Bax (1:1,000 dilution), anti-Bcl-2 (1:1,000 dilution), anti-Tom20 (1:1,000 dilution), anti-Drp1 (1:1,000 dilution), anti-cytochrome $c(1: 1,000$ dilution) (Cell Signaling Technology), anti-phospho-DRP1 (Ser637) (1:1,000 dilution), anti-Phospho-AKT (Ser473) (1:2,000 dilution) (Proteintech Group, Inc.). 
Mitochondrial fragmentation. Cells were cultured on slides and exposed to different treatments. The original complete medium was replaced with serum-free medium containing MitoTracker Red, and the cells were incubated at $37^{\circ} \mathrm{C}$ for $30 \mathrm{~min}$ and washed three times with PBS. Cells were then examined under an Echo-lab Revolve microscope.

PP2A phosphatase activity assays. PP2A phosphatase activity was measured with a PP2A Immunoprecipitation Phosphatase Assay Kit (Millipore). Treated cells were collected and added to RIPA lysis buffer, and malachite green was used to detect the free phosphate released by PP2A.

Immunofluorescence. Cells were cultured on slides and exposed to different treatments. Immunofluorescence analysis was performed following the addition of MitoTracker Red and DRP1 antibodies. Cells were then examined under an Echo-lab Revolve microscope.

Xenograft models. Twelve female BALB/c nude mice aged 6-8 weeks (Beijing Vital River Laboratory Animal Technology, Beijing, China) were placed in a standard microisolator in non-pathogenic conditions. Raised under the conditions of controlled temperature $\left(20-25^{\circ} \mathrm{C}\right)$ and light $(12$-h light/dark cycle), mice had free access to food and tap water, cages were ventilated, and wood chips were used as bedding, which was replaced every 3 days. The protocol was approved by the ethics committee of Jilin University National Health Research Institute in accordance with the National Institutes of Health Guidelines for the Care and Use of Laboratory Animals. The mice were injected subcutaneously into the left flank with $5 \times 10^{7}$ SKOV3 cells. When the average tumor volume of the animals was $30 \mathrm{~mm}^{3}$, they were randomly divided into four groups ( $n=3$ per group) as follows: Control group, 1, 2 and $3 \mathrm{mg} / \mathrm{kg}$ groups. Tumor volume was measured every two days and calculated using the formula: $0.5 \mathrm{x}$ length $\mathrm{x}$ width ${ }^{2}$. After 29 days of treatment, the mice were euthanized by cervical dislocation and tumors were excised for western blot analysis.

Statistical analysis. Data are expressed as mean \pm SD. Comparisons between groups were performed using one-way analysis of variance and Tukey's post hoc test. All the experiments were repeated three times. The data were analyzed by Chi-square and Spearman's rank correlation. All statistical analyses were performed using SPSS19.0 statistical software (SPSS Inc.). $\mathrm{P}<0.05$ was considered statistically significant.

\section{Results}

Polyphyllin VII affects cell viability and induces apoptosis in A2780 and SKOV3 cells. Fig. 1A shows the chemical structure of polyphyllin VII. To investigate the effect of polyphyllin VII on cell viability, we cultured cells with different concentrations $(0,1,2,3 \mu \mathrm{M})$ for $24 \mathrm{~h}$ and tested cell proliferation capacity with MTT. As shown in Fig. 1B and C, polyphyllin VII inhibited cell activity in a dose-dependent manner. Polyphyllin VII $(3 \mu \mathrm{M})$ significantly decreased the viability of A2780 and SKOV3 cells. These results suggested that polyphyllin VII can suppress the viability of different human ovarian cell lines. To further investigate the effect of polyphyllin VII on cell survival, we assessed apoptosis in ovarian cancer cell lines. A2780 and SKOV3 cells were cultured for $24 \mathrm{~h}$ with $0,1,2$ or $3 \mu \mathrm{M}$ Polyphyllin VII and flow cytometry analysis was performed with Annexin V-FITC/PI double staining. We found that Polyphyllin VII promoted the early and late apoptosis of A2780 and SKOV3 cells in a dose-dependent manner (Fig. 1D). Moreover, the expression of cleaved caspase-3 was significantly increased in the two cells (Fig. 1E). These results suggest that polyphyllin VII can induce apoptosis of human ovarian cancer cells.

Polyphyllin VII promotes mitochondrial dysfunction in A2780 and SKOV3 cells. A2780 and SKOV3 cells were stained with the ROS indicator DCFH-DA to evaluate the impact of polyphyllin VII on ROS production. The results showed that the ROS production in the two cell lines was increased in a dose-dependent manner after treatment with polyphyllin VII (Fig. 2A). In addition, polyphyllin VII decreased the mitochondrial membrane potential $(\Delta \psi \mathrm{m})$, which was confirmed by JC-1 probe application (Fig. 2B). With the increase in polyphyllin VII concentration, the intensity of green fluorescence increased gradually. As shown in Fig. 2C, we observed that compared with the control group, mitochondrial BAX expression in the polyphyllin VII-treated group was significantly upregulated, while mitochondrial BCL-2 expression was significantly reduced.

Polyphyllin VII intensifies DRPl-dependent mitochondrial fission. We used fluorescence microscopy to observe Mito-tracker red-stained mitochondrial morphology to evaluate changes in mitochondrial dynamics in A2780 and SKOV3 cells. As shown in Fig. 3A, after polyphyllin VII treatment at concentrations of 1,2 , and $3 \mu \mathrm{M}$, the proportion of mitochondrial fragmentation gradually increased compared with the control group. After treatment with $3 \mu \mathrm{M}$ polyphyllin VII, approximately $50 \%$ of the A2780 and SKOV3 cells were fragmented from the original grid-like mitochondrial structure. Additionally, we extracted mitochondria to detect protein expression by western blot analysis. DRP1 translocation to mitochondria was increased following polyphyllin VII treatment, and this phenomenon was most obvious at a concentration of $3 \mu \mathrm{M}$ (Fig. 3B-E). Mitochondrial localization of DRP1 is regulated by phosphorylation of DRP1 at Ser637. We found that the expression of phosphorylated DRP1 decreased with the increase in polyphyllin VII concentration. Studies have shown that DRP1 is a direct downstream molecule of AKT, and AKT can continuously regulate the activity of DRP1 (31,32). AKT-DRP1 interactions induce phosphorylation of DRP1, leading to mitochondrial division (Fig. 3F-H).

It has been shown that polyphyllin VII can inhibit tumor cell vitality via the PP2A/AKT pathway (29). We found that polyphyllin VII can downregulate phosphorylated AKT, and we hypothesized that polyphyllin VII can further regulate DRP1 through the regulation of AKT by PP2A. Therefore, to further examine the activation of PP2A upstream of AKT, we applied the PP2A immunoprecipitation phosphatase assay kit to detect the activity of PP2A, and found that the activity of PP2A was increased with increased polyphyllin VII concentration (Fig. 3I). 


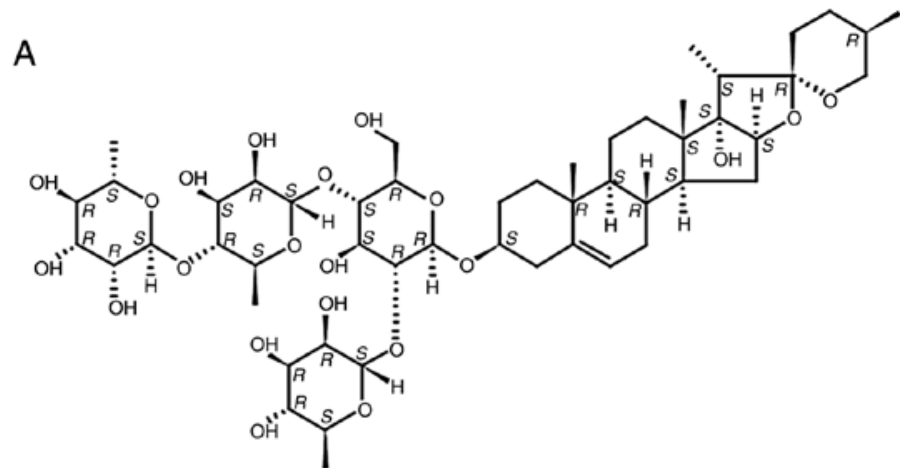
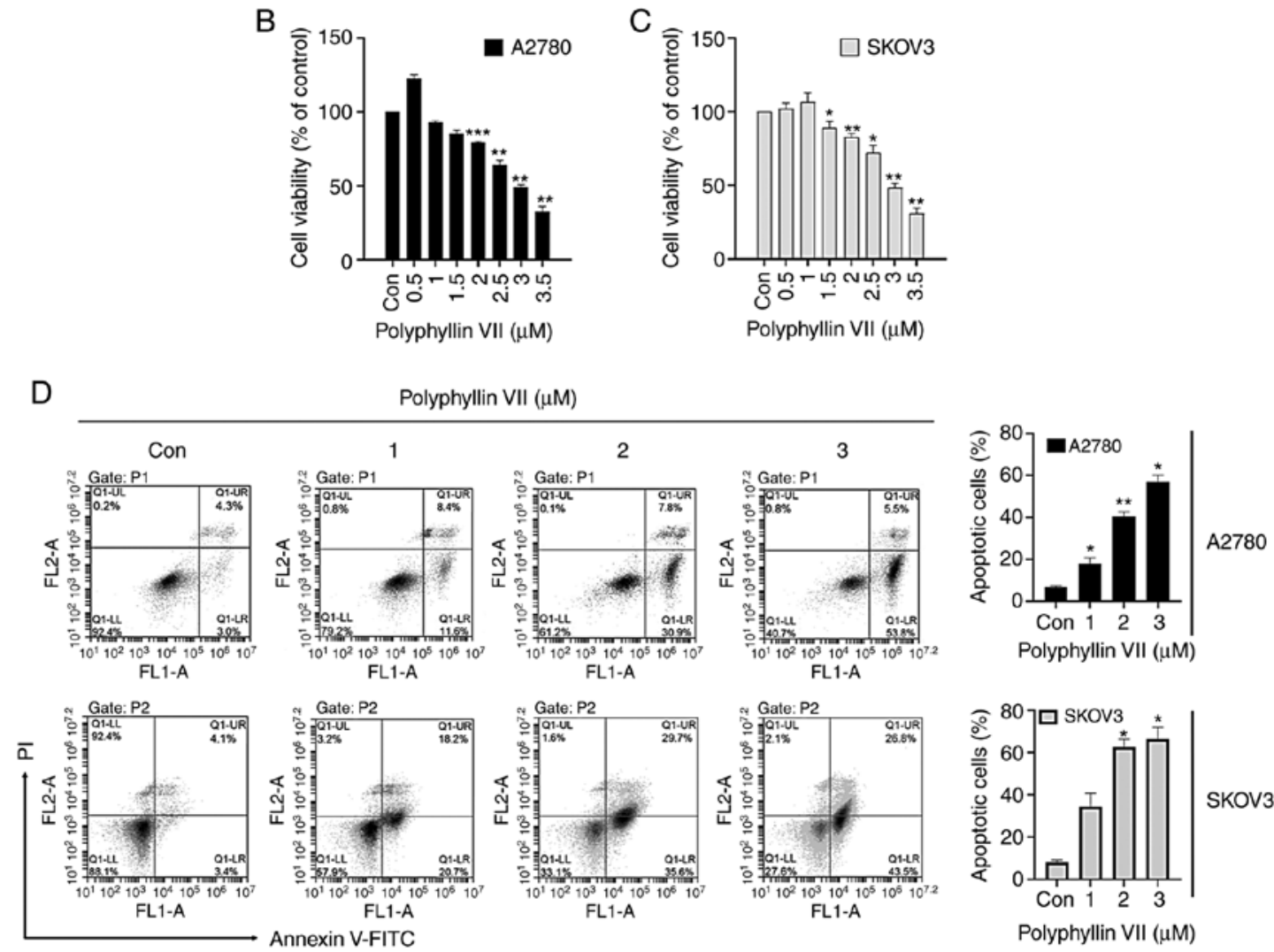

E
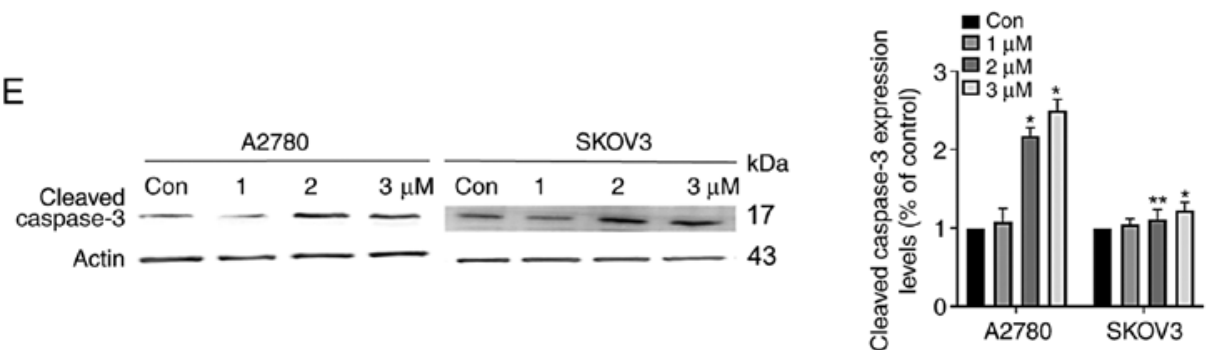

Figure 1. Polyphyllin VII affects A2780 and SKOV3 cells in a dose-dependent manner. (A) Chemical structure of polyphyllin VII. (B and C) A2780 and SKOV3 cells were treated with various concentrations of polyphyllin VII for $24 \mathrm{~h}$. The viability of A2780 and SKOV3 cells was measured by MTT assay. (D) Detection of A2780 and SKOV3 cell apoptotic fractions using Annexin V/propidium iodide staining. (E) Western blot analysis of cleaved caspase-3 (c-caspase-3) in A2780 and SKOV3 cells after polyphyllin VII treatment. Results are shown as mean \pm SD from three independent experiments performed in triplicate. ${ }^{*} \mathrm{P}<0.05,{ }^{* *} \mathrm{P}<0.01,{ }^{* * *} \mathrm{P}<0.001$, compared with the control $(0 \mu \mathrm{M})$.

Polyphyllin VII regulates the PP2A/AKT/DRP1 signaling axis in $A 2780$ and SKOV3 cells. To further confirm the role of the PP2A pathway in mediating the inhibition of polyphyllin VII on the proliferation of ovarian cancer cells, we introduced LB-100, a specific inhibitor of PP2A, to examine the changes of other molecules in the pathway. The results showed that LB-100 could reverse the increasing effect of DRP1 mitochondrial localization in the LB-100 group pretreated with polyphyllin VII (Fig. 4A and B), which was also confirmed by immunofluorescence (Fig. 4C). Cytochrome $c$ was significantly released in the cytoplasm after treatment with polyphyllin VII, which was inhibited by LB-100 (Fig. 4A and B). In addition, p-AKT and p-DRP1 expression decreased after polyphyllin VII treatment, which was 
A

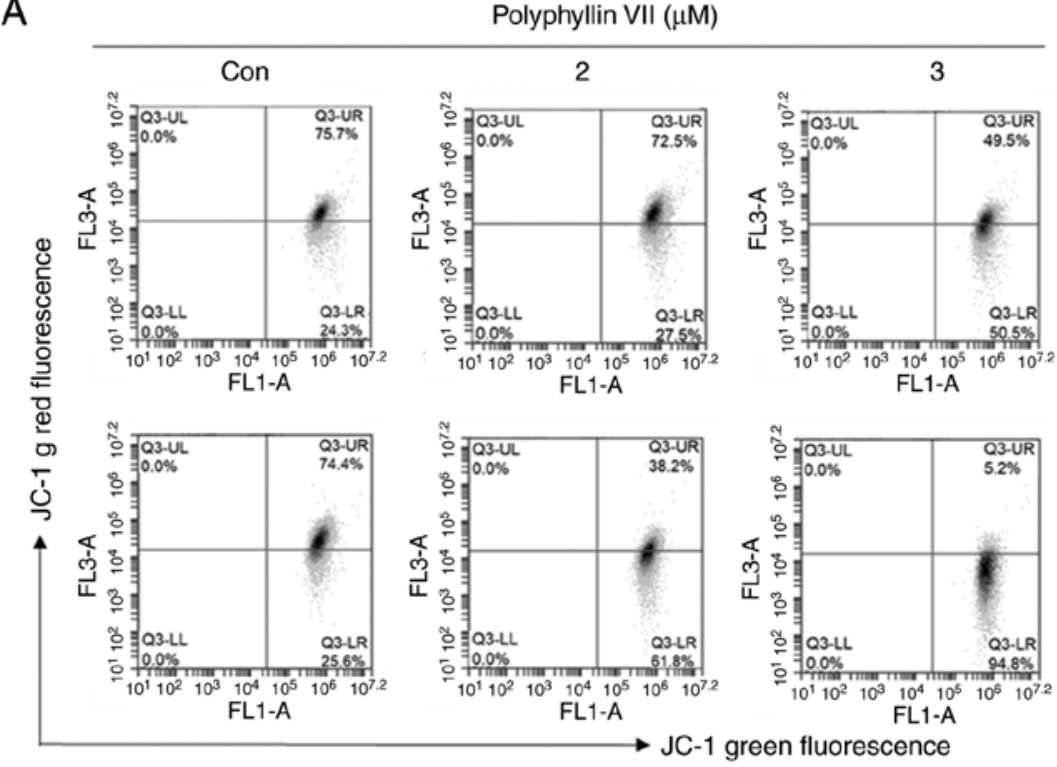

B
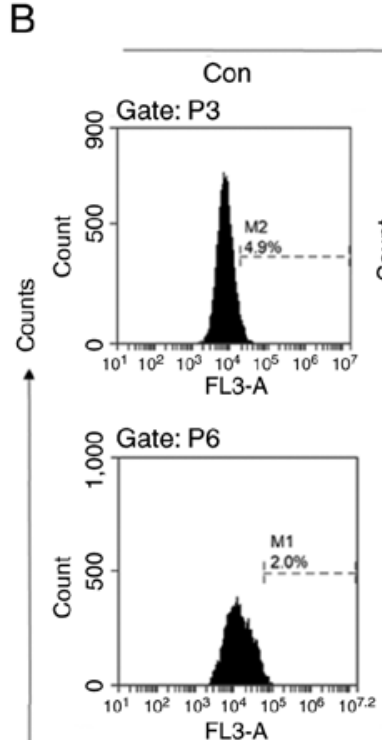

C

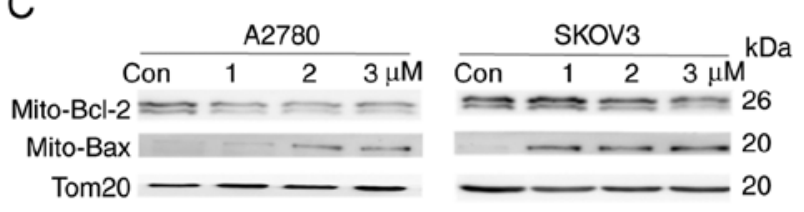

Polyphyllin VII $(\mu \mathrm{M})$
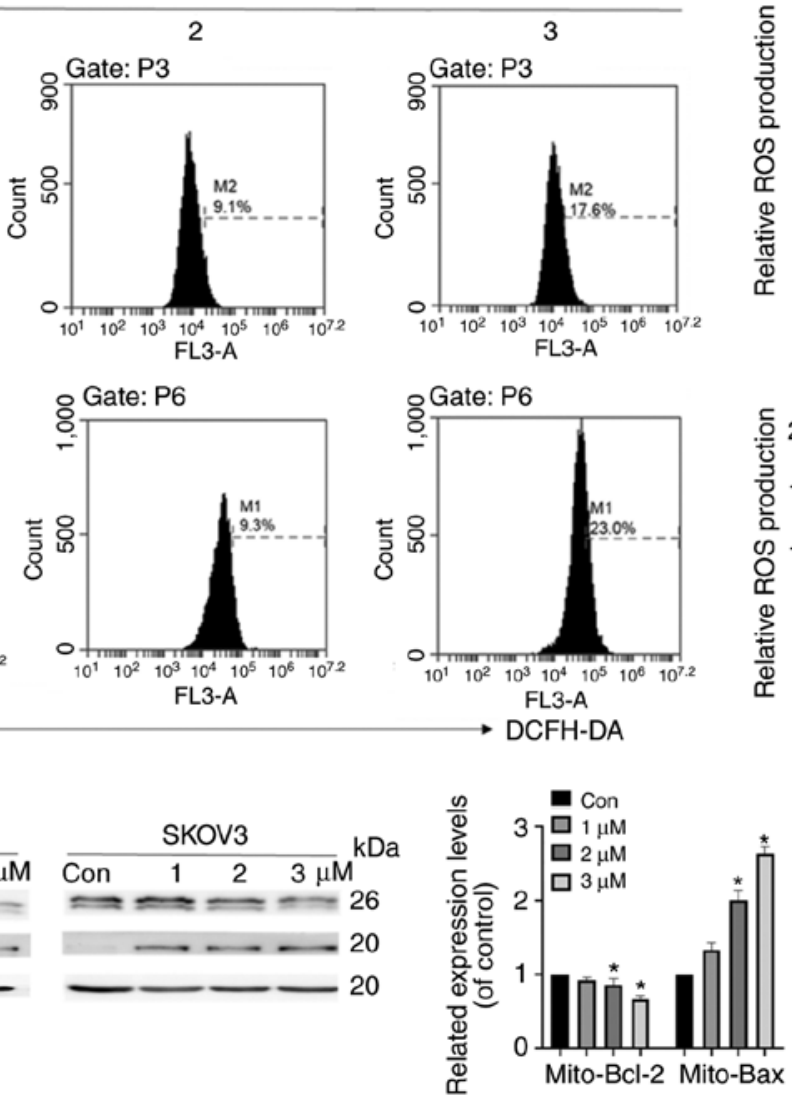

$\mathrm{A} 2780$

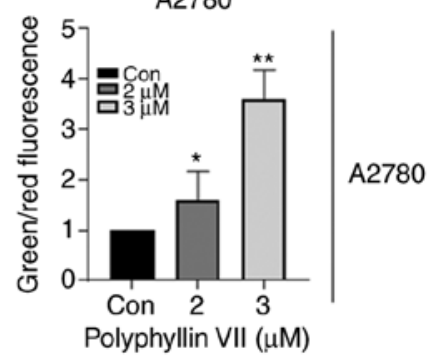

SKOV3

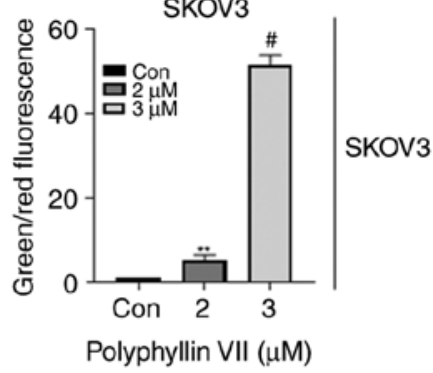

A2780
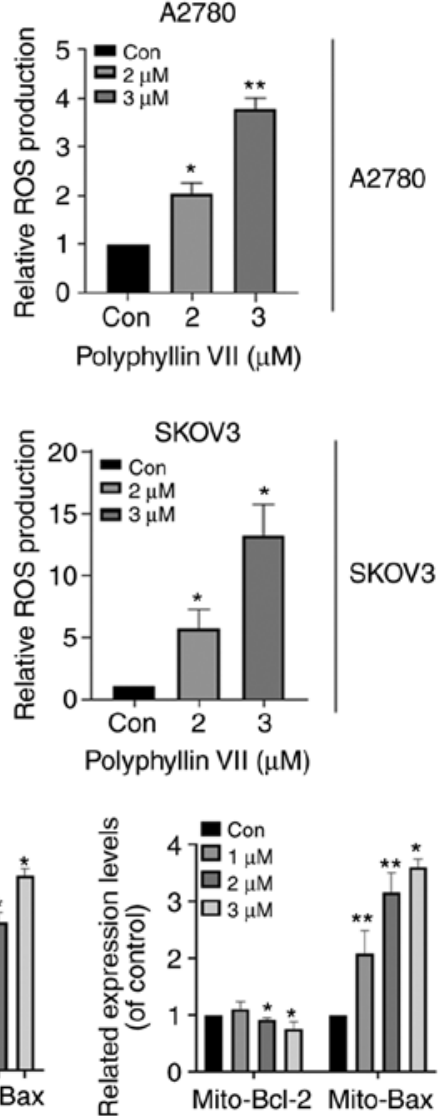

Figure 2. Polyphyllin VII promotes mitochondrial dysfunction in A2780 and SKOV3 cells. (A) DCFH-DA was used to evaluate ROS in A2780 and SKOV3 cells after polyphyllin VII treatment. (B) JC-1 was used to evaluate Dym in A2780 and SKOV3 cells after polyphyllin VII treatment. (C) Western blot analysis of BCL-2 and BAX in A2780 and SKOV3 cells after polyphyllin VII treatment. Results are shown as mean \pm SD from three independent experiments performed in triplicate. ${ }^{*} \mathrm{P}<0.05,{ }^{* *} \mathrm{P}<0.01,{ }^{\#} \mathrm{P}<0.001$, compared with the control $(0 \mu \mathrm{M})$.

largely changed by LB-100 pretreatment (Fig. 4D). As shown in Fig. 4E, LB-100 can significantly inhibit PP2A activity effectively, and it can reverse the increase of PP2A activity induced by polyphyllin VII. Based on the above results, we concluded that polyphyllin VII changes DRP1 localization in mitochondrial by regulating the PP2A/AKT/DRP1 signaling axis, thereby promoting mitochondrial division.
Polyphyllin VII inhibits tumor growth in vivo. To study the effects of polyphyllin VII treatment in vivo, we constructed a xenograft tumor model in nude mice (Fig. 5A and B). Compared with the control, the tumor growth was significantly inhibited, and the weight of nude mice was significantly decreased (Fig. 5C and D). The ratio of BAX/BCL-2 was upregulated and the expression of cleaved caspase- 3 was 
A Polyphyllin VII $(\mu \mathrm{M})$
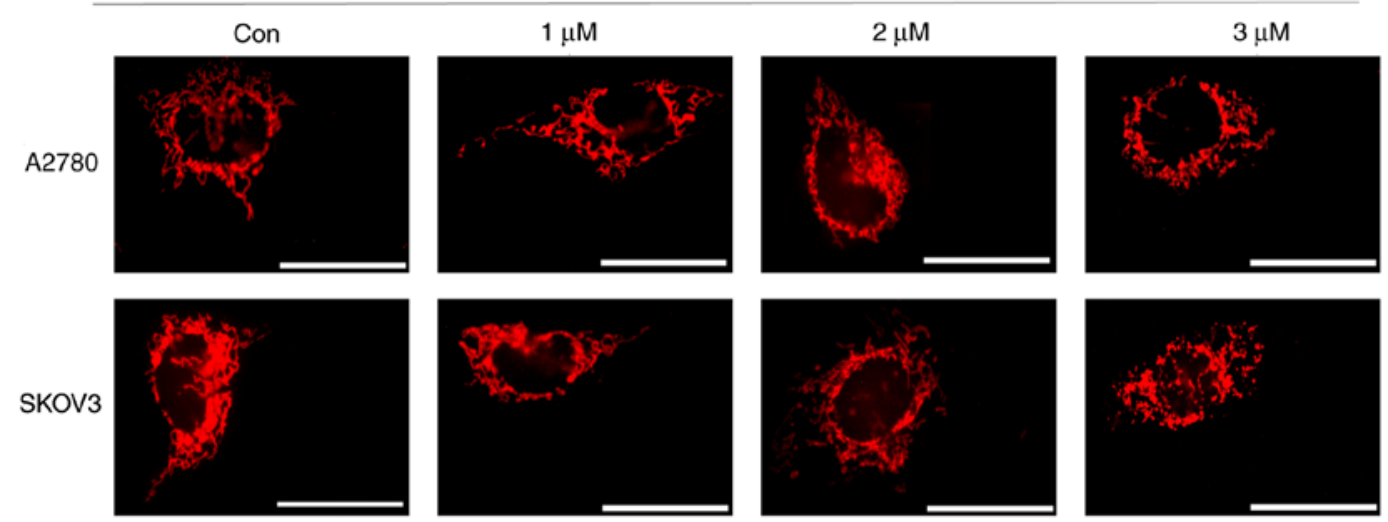

B
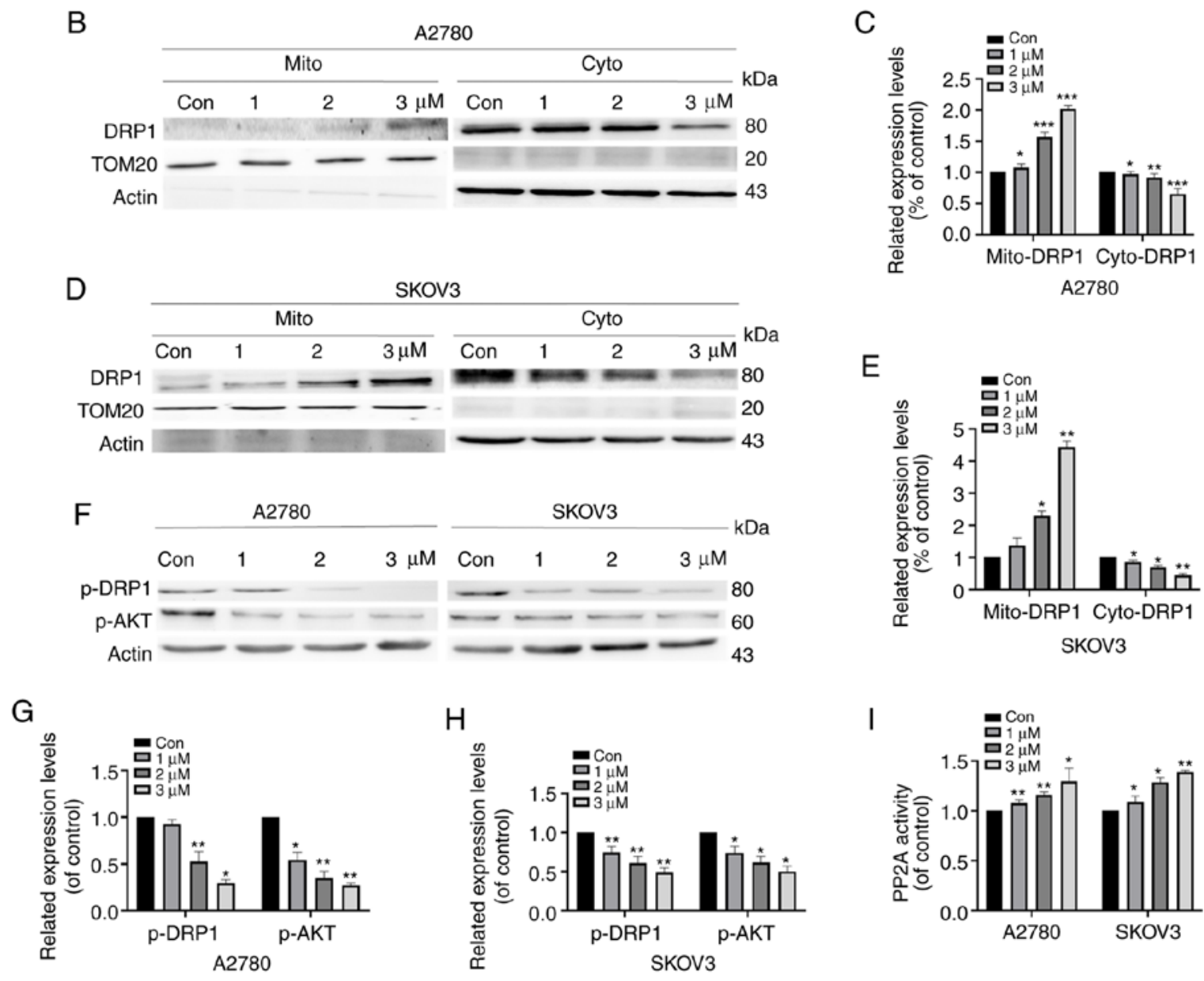

Figure 3. Polyphyllin VII intensifies DRP1-dependent mitochondrial fission. (A) A2780 and SKOV3 cells were treated with various concentrations of polyphyllin VII for $24 \mathrm{~h}$. Mito-tracker red fluorescence was used to evaluate mitochondrial fission, indicating the mitochondria in terms of the percentage of debris. Scale bar, $70 \mu \mathrm{m}$. (B and D) Polyphyllin VII treatment reduced DRP1 translocation to the mitochondria. Mitochondria were extracted for western blot analysis. (C and E) Quantification of Fig. 3B and D. (F) Western blot analysis of p-DRP1 and p-AKT in A2780 and SKOV3 cells after polyphyllin VII treatment. Polyphyllin VII treatment increased DRP1 and AKT phosphorylation. (G and H) Quantification of Fig. 3F. (I) After A2780 and SKOV3 were treated with polyphyllin VII at different concentrations for $24 \mathrm{~h}$, PP2A activity assay was performed. Results are shown as mean \pm SD from three independent experiments performed in triplicate. ${ }^{*} \mathrm{P}<0.05,{ }^{* *} \mathrm{P}<0.01,{ }^{* * *} \mathrm{P}<0.001$, compared with the control $(0 \mu \mathrm{M})$.

significantly increased (Fig. 5C). These results further suggest that polyphyllin VII is a potential treatment for ovarian cancer.

\section{Discussion}

Although the anti-tumor effect of polyphyllin VII on various tumors including ovarian cancer has been widely studied $(5,6,29)$, the changes in mitochondrial function and dynamics are still poorly understood. To the best of our knowledge, this study is the first to report that regulation of the PP2A/AKT/DRP1 axis promotes mitochondrial division and inhibits the proliferation of human ovarian cancer cells. We selected the most commonly used ovarian cancer cell lines A2780 and SKOV3 to study the anticancer effects of polyphyllin VII. Although A2780 and SKOV3 we used may not represent the most common type of high-grade serous ovarian 
A

A
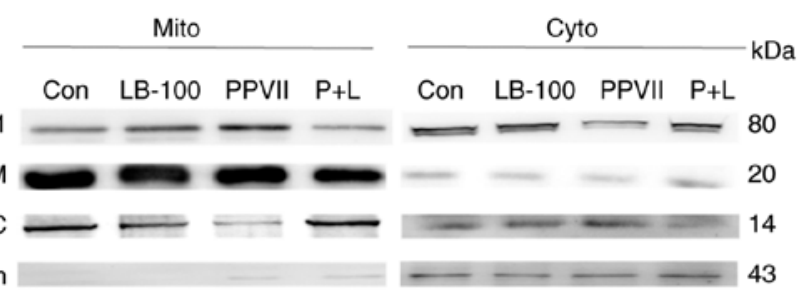

B
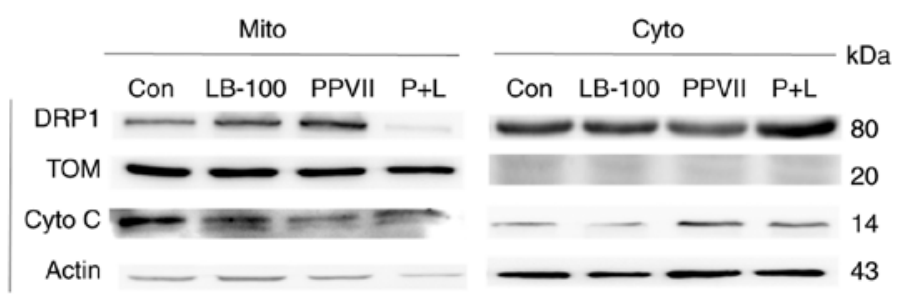

C
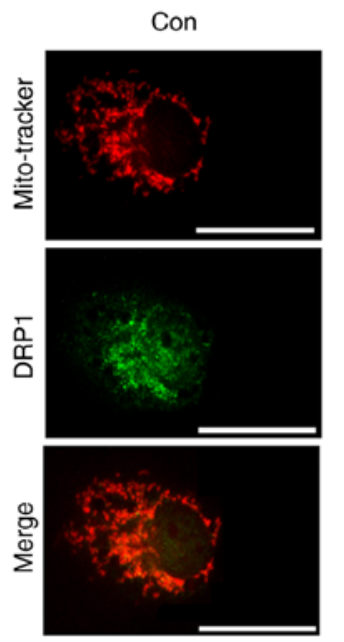

D

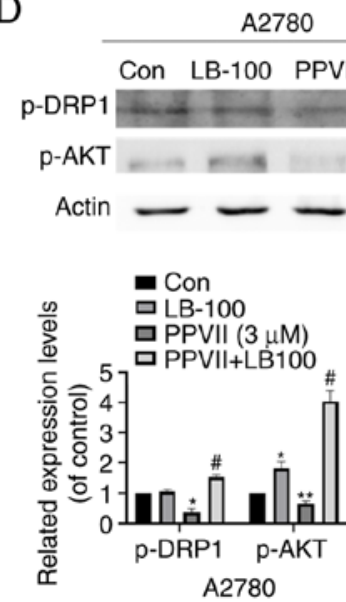

LB-100
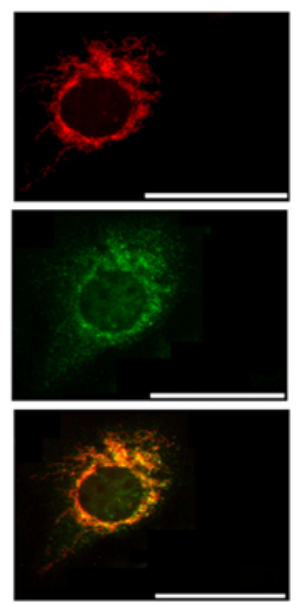
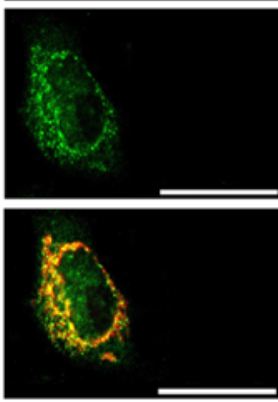

SKOV3

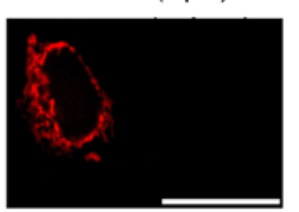

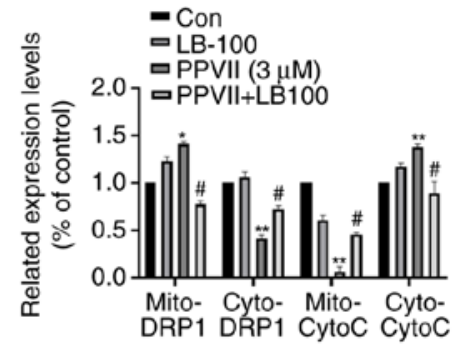

A2780

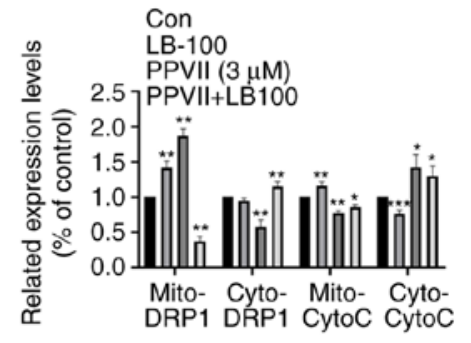

SKOV3

$\mathrm{P}+\mathrm{L}$
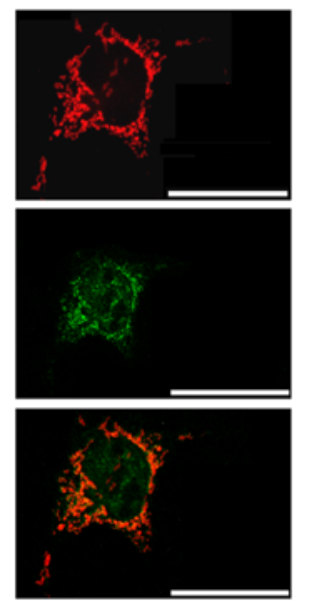


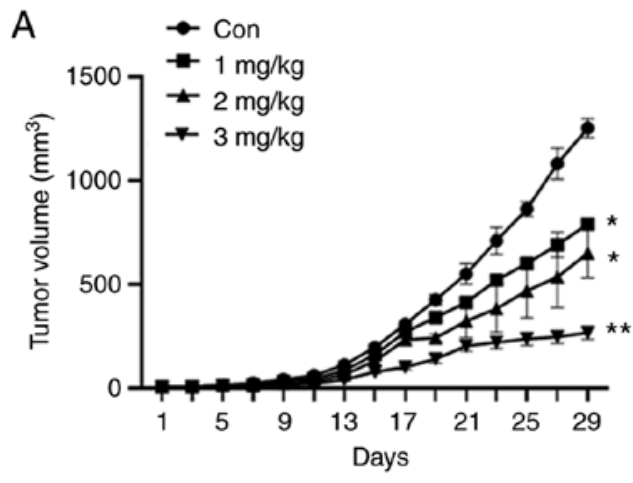

C

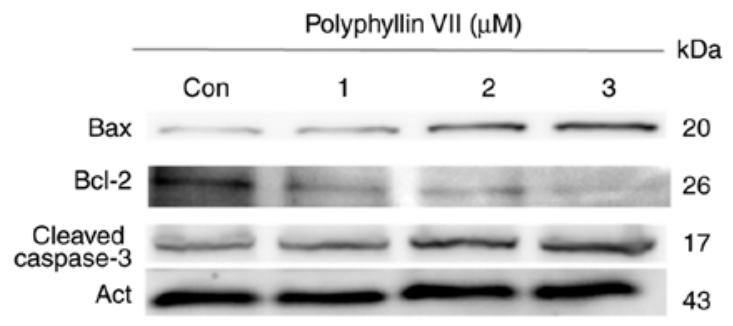

B

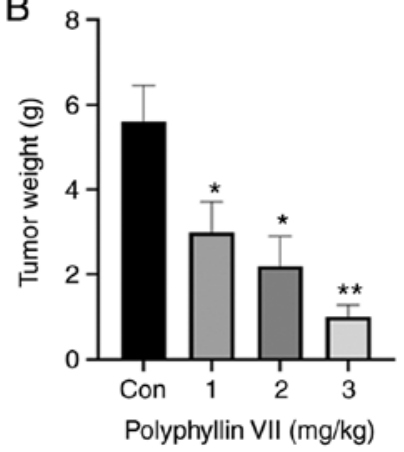

D

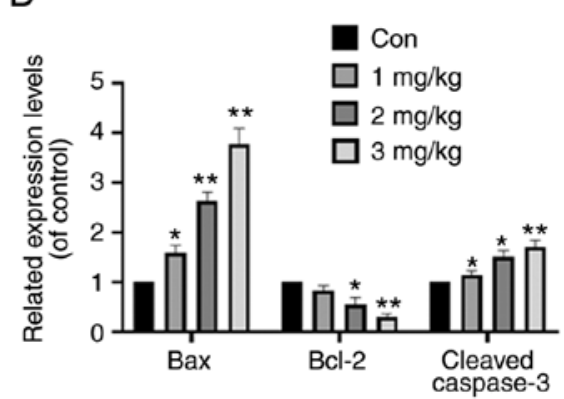

Figure 5. Polyphyllin VII inhibits tumor growth in vivo. SKOV3 cells were subcutaneously implanted into nude mice. Each treatment group consisted of three mice treated with polyphyllin VII (1 mg/kg body weight) for 31 days. (A) Tumor growth curves were constructed based on tumor size, which was measured every 2 days for each treatment condition. (B) Wet weights of tumors were determined at autopsy. Columns represent mean of three tumors. ${ }^{*} \mathrm{P}<0.05$ compared with the control group; ${ }^{* *} \mathrm{P}<0.01$ compared with control group. (C) Representative image of tumor from each group. (D and E) Western blot analysis of c-caspase-3, BCL-2, and BAX expression after treatments.

control their homeostasis, among which constant fission and fusion are important dynamics (33). Imbalances in mitochondrial division and fusion often lead to changes in mitochondrial structure and dysfunction. Our study found that ovarian cells generated substantial amounts of ROS, which was accompanied by Dym collapse after polyphyllin VII treatment. Therefore, we aimed to determine whether this mitochondrial dysfunction was related to increased mitochondrial division, and whether it was a good point from which to start exploring new ways to combat cancer. DRP1 mediates mitochondrial division, and can translocate from the cytoplasm to the mitochondria and contract the mitochondria so that mitochondrial division results in two independent organelles. Dysfunctional mitochondria may lose their ability to fuse by activating the mitochondrial division mechanism, preventing the damaged mitochondria from re-entering the healthy mitochondrial network (34). There is increasing evidence that post-translational modification of DRP1 is an important mechanism for regulating its function (14). Under the stimulation of apoptosis, the mitochondrial network collapses into a small spherical structure, and the BCL-2 family has an important role in regulating mitochondrial morphology (35). During the process of cell apoptosis, BAX activation co-locates BAX and DRP1 at mitochondrial sites where division occurs (16), and DRP1 stably binds to the mitochondrial outer membrane, leading to mitochondrial fragmentation (17-19). It has been shown that DRP1 activates and oligomerizes BAX by promoting the formation of an intermediate, creating a pore and leading to permeability of the mitochondrial outer membrane (MOM) (36). Mitochondrial division is accompanied by MOM permeability, mitochondrial crest disorder, and the release of cytochrome $c(37,38)$. Our results showed that polyphyllin VII treatment could increase DRP1 localization in mitochondria, upregulate the mitochondrial BAX/BCL-2 ratio, and increase the release of cytochrome $c$. Phosphorylated DRP1 (Ser637) can regulate DRP1 localization in mitochondria and induce mitochondrial division. Our study showed that polyphyllin VII can dephosphorylate DRP1, thus recruiting DRP1 to mitochondria. The process of mitochondrial fission runs through cell proliferation and is affected by various kinases. AKT, which directly regulates DRP1, is a serine/threonine kinase that has a significant role in cell proliferation and survival $(30,32)$.

Protein phosphatase A (PP2A) can regulate multiple cellular processes by dephosphorylating many critical cellular molecules such as PKC, AKT, $\beta$-catenin, and c-Myc, and plays an important role as a tumor suppressor. PP2A has been shown to regulate various biological processes in humans, such as cell DNA replication, transcription, translation, cell cycle, cell proliferation, apoptosis, and migration, and has also been shown to regulate cell transformation and cancer (39-42). PP2A is deactivated in various malignancies, including breast (43), ovarian (44), cervical (45), and lung carcinoma (46). Partial loss of PP2A phosphatase activity may lead to unrestricted carcinogenic kinase activity, triggering carcinogenic signals. Reactivation of PP2A is an effective way to antagonize cancer, and our study showed that polyphyllin VII can activate PP2A and inactivate AKT and DRP1. The application of PP2A inhibitors further confirmed that the mechanism of action of polyphyllin VII is through the PP2A/AKT/DRP1 axis.

To summarize, the aim of this study was to explore new drugs that promote cancer cell apoptosis by regulating mitochondrial dynamics and to identify new therapies to prevent and treat cancer progression. Our results showed that 
polyphyllin VII can increase Drpl localization in mitochondria, thus exacerbating mitochondrial division and leading to apoptosis. We also found that the above changes are due to the regulation of the PP2A/AKT/DRP1 signaling axis. However, further studies are required to verify these results.

\section{Acknowledgements}

Not applicable.

\section{Funding}

The present study was funded by Natural Science Foundation of Jilin Province (no. 20180101134C).

\section{Availability of data and materials}

The datasets used and/or analyzed during the present study are available from the corresponding author on reasonable request.

\section{Authors' contributions}

LZ contributed the experimental design and wrote the draft. ZL and XD collected and sorted out relevant literature, conducted experiments and collected data. JW and LS carried out data analysis and revised the manuscript. LF and YZ made substantial contributions to the interpretation and analysis of the data, drafting the study and revising it critically for important intellectual content. All authors have read and reviewed the final manuscript.

\section{Ethics approval and consent to participate}

The protocol was approved by the ethics committee of Jilin University National Health Research Institute in accordance with National Institutes of Health Guidelines for the Care and Use of Laboratory Animals.

\section{Patient consent for publication}

Not applicable.

\section{Competing interests}

The authors declare that they have no competing interests.

\section{References}

1. Zhang C, Li C, Jia X, Wang K, Tu Y, Wang R, Liu K, Lu T and He C: In vitro and in vivo anti-inflammatory effects of polyphyllin VII through downregulating MAPK and NF-kB pathways. Molecules 24: 875, 2019.

2. Liu Z, Li N, Gao W, Man S, Yin S and Liu C: Comparative study on hemostatic, cytotoxic and hemolytic activities of different species of Paris L. J Ethnopharmacol 142: 789-794, 2012.

3. Zhang XF, Cui Y, Huang JJ, Zhang YZ, Nie Z, Wang LF, Yan BZ, Tang YL and Liu Y: Immuno-stimulating properties of diosgenyl saponins isolated from Paris polyphylla. Bioorg Med Chem Lett 17: 2408-2413, 2007.

4. Al Sawah E, Marchion DC, Xiong Y, Ramirez IJ, Abbasi F, Boac BM, Bush SH, Bou Zgheib N, McClung EC, Khulpateea BR, et al: The Chinese herb polyphyllin D sensitizes ovarian cancer cells to cisplatin-induced growth arrest. J Cancer Res Clin Oncol 141: 237-242, 2015
5. Liu X, Sun Z, Deng J, Liu J, Ma K, Si Y, Zhang T, Feng T, Liu Y and Tan Y: Polyphyllin I inhibits invasion and epithelial-mesenchymal transition via CIP2A/PP2A/ERK signaling in prostate cancer. Int J Oncol 53: 1279-1288, 2018.

6. He J, Yu S, Guo C, Tan L, Song X, Wang M, Wu J, Long Y, Gong D, Zhang R, et al: Polyphyllin I induces autophagy and cell cycle arrest via inhibiting PDK1/Akt/mTOR signal and downregulating cyclin $\mathrm{B} 1$ in human gastric carcinoma HGC-27 cells. Biomed Pharmacother 117: 109189, 2019.

7. Hong F, Gu W, Jiang J, Liu X and Jiang H: Anticancer activity of polyphyllin I in nasopharyngeal carcinoma by modulation of lncRNA ROR and P53 signalling. J Drug Target 27: 806-811, 2019.

8. Lin LT, Uen WC, Choong CY, Shi YC, Lee BH, Tai CJ and Tai CJ: Paris polyphylla inhibits colorectal cancer cells via inducing autophagy and enhancing the efficacy of chemotherapeutic drug doxorubicin. Molecules 24: 2102, 2019.

9. Yang C, Cai H and Meng X: Polyphyllin D induces apoptosis and differentiation in K562 human leukemia cells. Int Immunopharmacol 36: 17-22, 2016.

10. Liu Y, Dong X, Wang W, You L, Yin X, Yang C, Sai N, Leng X and Ni J: Molecular mechanisms of apoptosis in HepaRG cell line induced by polyphyllin VI via the fas death pathway and mitochondrial-dependent pathway. Toxins (Basel) 10: 201, 2018.

11. Zorov DB, Juhaszova M and Sollott SJ: Mitochondrial reactive oxygen species (ROS) and ROS-induced ROS release. Physiol Rev 94: 909-950, 2014.

12. Adam-Vizi V and Chinopoulos C: Bioenergetics and the formation of mitochondrial reactive oxygen species. Trends Pharmacol Sci 27: 639-645, 2006.

13. Choudhury AR and Singh KK: Mitochondrial determinants of cancer health disparities. Semin Cancer Biol 47: 125-146, 2017.

14. Tilokani L, Nagashima S, Paupe V and Prudent J: Mitochondrial dynamics: Overview of molecular mechanisms. Essays Biochem 62: 341-360, 2018.

15. Cannino G, Ciscato F, Masgras I, Sanchez-Martin C and Rasola A: Metabolic plasticity of tumor cell mitochondria. Front Oncol 8: 333, 2018.

16. Karbowski M, Lee YJ, Gaume B, Jeong SY, Frank S, Nechushtan A, Nechushtan A, Santel A, Fuller M, Smith CL and Youle RJ: Spatial and temporal association of Bax with mitochondrial fission sites, Drp1, and Mfn2 during apoptosis. J Cell Biol 159: 931-938, 2002

17. Karbowski M, Arnoult D, Chen H, Chan DC, Smith CL and Youle RJ: Quantitation of mitochondrial dynamics by photolabeling of individual organelles shows that mitochondrial fusion is blocked during the Bax activation phase of apoptosis. J Cell Biol 164: 493-499, 2004.

18. Brooks C, Wei Q, Feng L, Dong G, Tao Y, Mei L, Xie ZJ and Dong Z: Bak regulates mitochondrial morphology and pathology during apoptosis by interacting with mitofusins. Proc Natl Acad Sci USA 104: 11649-11654, 2007.

19. Wasiak S, Zunino R and McBride HM: Bax/Bak promote sumoylation of DRP1 and its stable association with mitochondria during apoptotic cell death. J Cell Biol 177: 439-450, 2007.

20. Hasnat M, Yuan Z, Ullah A, Naveed M, Raza F, Baig MMFA, Khan A, Xu D, Su Y, Sun L, et al: Mitochondria-dependent apoptosis in triptolide-induced hepatotoxicity is associated with the Drp1 activation. Toxicol Mech Methods 30: 124-133, 2020.

21. Li G, Zhou J, Budhraja A, Hu X, Chen Y, Cheng Q, Liu L, Zhou T, Li P, Liu E and Gao N: Mitochondrial translocation and interaction of cofilin and Drp1 are required for erucin-induced mitochondrial fission and apoptosis. Oncotarget 6: 1834-1849, 2005.

22. O'Connor CW, Perl A, Leonard D, Sangodkar J and Narla G: Therapeutic targeting of PP2A. Int J Biochem Cell Biol 96: 182-193, 2018.

23. Raman D and Pervaiz S: Redox inhibition of protein phosphatase PP2A: Potential implications in oncogenesis and its progression. Redox Biol 27: 101105, 2019.

24. Rincon R, Cristobal I, Zazo S, Arpi O, Menendez S, Manso R, Lluch A, Eroles P, Rovira A, Albanell J, et al: PP2A inhibition determines poor outcome and doxorubicin resistance in early breast cancer and its activation shows promising therapeutic effects. Oncotarget 6: 4299-4314, 2015.

25. Sugiyama M, Imai A, Furui T and Tamaya T: Independent action of serine/threonine protein phosphatase in ovarian cancer plasma membrane and cytosol during gonadotropin-releasing hormone stimulation. Oncol Rep 10: 1885-1889, 2003. 
26. Zheng HY, Shen FJ, Tong YQ and Li Y: PP2A Inhibits cervical cancer cell migration by dephosphorylation of p-JNK, p-p38 and the p-ERK/MAPK signaling pathway. Curr Med Sci 38: 115-123, 2018

27. Nader CP, Cidem A, Verrills NM and Ammit AJ: Protein phosphatase 2A (PP2A): A key phosphatase in the progression of chronic obstructive pulmonary disease (COPD) to lung cancer. Respir Res 20: 222, 2019.

28. Perrotti D and Neviani P: Protein phosphatase 2A: A target for anticancer therapy. Lancet Oncol 14: e229-e238, 2013.

29. Zhang Y, Huang P, Liu X, Xiang Y, Zhang T, Wu Y, Xu J, Sun Z, Zhen W, Zhang L, et al: Polyphyllin I inhibits growth and invasion of cisplatin-resistant gastric cancer cells by partially inhibiting CIP2A/PP2A/Akt signaling axis. J Pharmacol Sci 137: 305-312, 2018.

30. Kim DI, Lee KH, Gabr AA, Choi GE, Kim JS, Ko SH and Han HJ: A $\beta$-induced Drp1 phosphorylation through Akt activation promotes excessive mitochondrial fission leading to neuronal apoptosis. Biochim Biophys Acta 1863: 2820-2834, 2016.

31. Zhou X, Wang HY, Wu B, Cheng CY, Xiao W, Wang ZZ, Yang YY, Li P and Yang H: Ginkgolide K attenuates neuronal injury after ischemic stroke by inhibiting mitochondrial fission and GSK-3 $\beta$-dependent increases in mitochondrial membrane permeability. Oncotarget 8: 44682-44693, 2017.

32. Tao A, Xu X, Kvietys P, Kao R, Martin C and Rui T: Experimental diabetes mellitus exacerbates ischemia/reperfusion-induced myocardial injury by promoting mitochondrial fission: Role of down-regulation of myocardial Sirt1 and subsequent Akt/Drp1 interaction. Int J Biochem Cell Biol 105: 94-103, 2018.

33. Samanta K, Douglas S and Parekh AB: Mitochondrial calcium uniporter MCU supports cytoplasmic $\mathrm{Ca} 2+$ oscillations, store-operated $\mathrm{Ca}^{2+}$ entry and $\mathrm{Ca}^{2+}$-dependent gene expression in response to receptor stimulation. PLoS One 9: e101188, 2014.

34. Simula L, Nazio F and Campello S: The mitochondrial dynamics in cancer and immune-surveillance. Semin Cancer Biol 47 29-42, 2017.

35. Martinou JC and Youle RJ: Mitochondria in apoptosis: Bcl-2 family members and mitochondrial dynamics. Dev Cell 21: 92-101, 2011.

36. Montessuit S, Somasekharan SP, Terrones O, LuckenArdjomande S, Herzig S, Schwarzenbacher R, Manstein DJ, Bossy-Wetzel E, Basañez G, Meda P and Martinou JC: Membrane remodeling induced by the dynamin-related protein Drp1 stimulates bax oligomerization. Cell 142: 889-901, 2010.
37. Lee YJ, Jeong SY, Karbowski M, Smith CL and Youle RJ: Roles of the mammalian mitochondrial fission and fusion mediators Fis1, Drp1, and Opal in apoptosis. Mol Biol Cell 15: 5001-5011, 2004.

38. Frank S, Gaume B, Bergmann-Leitner ES, Leitner WW, Robert EG, Catez F, Smith CL and Youle RJ: The role of dynamin-related protein 1, a mediator of mitochondrial fission, in apoptosis. Dev Cell 1: 515-525, 2001.

39. Alberts AS, Thorburn AM, Shenolikar S, Mumby MC and Feramisco JR: Regulation of cell cycle progression and nuclear affinity of the retinoblastoma protein by protein phosphatases. Proc Natl Acad Sci USA 90: 388-392, 1993.

40. Glenn GM and Eckhart W: Mutation of a cysteine residue in polyomavirus middle $\mathrm{T}$ antigen abolishes interactions with protein phosphatase $2 \mathrm{~A}$, pp $60 \mathrm{c}$-src, and phosphatidylinositol-3 kinase, activation of c-fos expression, and cellular transformation. J Virol 67: 1945-1952, 1993.

41. Ronne H, Carlberg M, Hu GZ and Nehlin JO: Protein phosphatase 2A in saccharomyces cerevisiae: Effects on cell growth and bud morphogenesis. Mol Cell Biol 11: 4876-4884, 1991.

42. Wlodarchak N and Xing Y: PP2A as a master regulator of the cell cycle. Crit Rev Biochem Mol Biol 51: 162-184, 2016.

43. Tseng LM, Liu CY, Chang KC, Chu PY, Shiau CW and Chen KF: CIP2A is a target of bortezomib in human triple negative breast cancer cells. Breast Cancer Res 14: R68, 2012.

44. Bockelman C, Lassus H, Hemmes A, Leminen A, Westermarck J, Haglund C, Bützow R and Ristimäki A: Prognostic role of CIP2A expression in serous ovarian cancer. Br J Cancer 105: 989-995, 2011.

45. Shih IeM, Panuganti PK, Kuo KT, Mao TL, Kuhn E, Jones S, Velculescu VE, Kurman RJ and Wang TL: Somatic mutations of PPP2R1A in ovarian and uterine carcinomas. Am J Pathol 178: $1442-1447,2011$

46. Xu P, Xu XL, Huang Q, Zhang ZH and Zhang YB: CIP2A with survivin protein expressions in human non-small-cell lung cancer correlates with prognosis. Med Oncol 29: 1643-1647, 2012.

This work is licensed under a Creative Commons Attribution-NonCommercial-NoDerivatives 4.0 International (CC BY-NC-ND 4.0) License. 